\title{
A low complex and efficient coexistence approach for non-coherent multiband impulse radio UWB
}

\author{
Hanns-Ulrich Dehner*, Micha Linde*, Rainer Moorfeld ${ }^{\ddagger}$, Holger Jäkel*, Dennis Burgkhardt*, \\ Friedrich Jondral*, and Adolf Finger ${ }^{\ddagger}$ \\ *Institut für Nachrichtentechnik, Universität Karlsruhe (TH), 76128 Karlsruhe, Germany \\ ${ }^{\ddagger}$ Communications Laboratory, Dresden University of Technology, 01062 Dresden, Germany \\ Email: \{dehner, linde, jaekel, burgkhardt, fj\}@int.uni-karlsruhe.de, \{moorfeld, finger\}@ifn.et.tu-dresden.de
}

\begin{abstract}
When bringing a high data rate multiband impulse radio UWB system to the market it has to coexist with other already existing UWB technologies such as multiband OFDM UWB [1]. This paper analyzes the interference impact of a multiband OFDM UWB system on a non-coherent multiband impulse radio UWB system. It is shown that the impact can be so dominant that communication within the impulse based multiband UWB system gets worse. For this reason a static as well as a dynamic coexistence approach are considered. The dynamic approach uses an efficient, robust and easy to realize "pixel" based interference detection algorithm in conjunction with an adapted bandplan array. In comparison to the static approach, the dynamic coexistence approach allows higher data rates during data transmission phases without significant losses in bit error rate performance.
\end{abstract}

Index Terms-Multiband UWB, impulse radio UWB, energy detection, coexistence

\section{INTRODUCTION}

Ultra Wideband (UWB) is a promising candidate for future high data rate wireless communications over short distances. It operates as an unlicensed radio technology using an occupied $-10 \mathrm{~dB}$ bandwidth which has to be greater than $20 \%$ of the arithmetic center frequency or greater than $500 \mathrm{MHz}$ [2]. Among other things UWB supports a low power, low cost and low complexity realization in conjunction with high system capacity, scalability and flexibility. Thereby, a very easy to realize monoband UWB transmission technique is carrierless impulse radio UWB [3]. Extremely short pulses are sent, whereas signal energy is spread over several gigahertz. To increase system capacity this method can be extended to a non-coherent multiband impulse radio UWB (MIR-UWB) approach [4], [5], [6], whose single user performance was analyzed in [7].

However, as UWB is an underlying radio technology, it has to coexist with a large number of licensed and unlicensed systems, operating in the same frequency range of $f_{1}=3.1 \mathrm{GHz}$ to $f_{\mathrm{u}}=10.6 \mathrm{GHz}$. In this context, one important unlicensed broadband interference source for indoor environments could be multiband OFDM UWB (MB-OFDM UWB) according to IEEE 802.15. That's why further analysis of MIR-UWB system's broadband interference coexistence behavior is necessary.

This paper presents and compares two simple and efficient coexistence strategies for a non-coherent MIR-UWB system. In a static coexistence approach, subbands of the MIR-UWB system, which could be possibly interfered, are completely deactivated before any data transmission occurs. In contrast, a more dynamic coexistence strategy is based on a system specific channel estimation which is described in [5] in conjunction with a "pixel" based reliable and robust interference detection algorithm. Thereby, without any adaptation of the MIR-UWB system parameters, subbands are used for data transmission in accordance to an adapted twodimensional bandplan array which considers interference-free time-frequency gaps.

The remainder of the paper is organized as follows: Section II gives a short introduction into the MIR-UWB system architecture. The considered MB-OFDM UWB interference source as well as its impact on the MIR-UWB system is described in Section III. This leads to Section IV in which a low complex and efficient coexistence approach is presented. Finally Section V gives a summary as well as an outlook.

\section{MIR-UWB SYSTEM}

\section{A. Transmitter Structure}

On the MIR-UWB transmitter side a filter bank approach [7] is used to generate $N_{\text {sub }}$ subband pulses. An ultra short pulse is regularly emitted by a pulse generator to the input of an analog band-pass filter bank, consisting of $N_{\text {sub }}$ adjacent bandpass filters with filter order $n_{\mathrm{f}}$ and $3 \mathrm{~dB}$ cutoff frequencies $f_{c_{\mathrm{i}}} \pm B_{\text {eff }} / 2$. Thereby, $B_{\text {eff }}=B_{\text {sub }}-B_{\text {off }}$ stands for the effective available subband bandwidth, $B_{\text {sub }}=\left(f_{\mathrm{u}}-f_{1}\right) / N_{\text {sub }}$ for the theoretical available subband bandwidth, $B_{\text {off }}$ for the frequency offset to reduce intersubband interference between adjacent filters and $f_{c_{\mathrm{i}}}$ for the filters' center frequencies. Then, the filter bank output consists of $N_{\text {sub }}$ specific subband pulses. Simultaneously, uniformly distributed bits $b \in\{0,1\}$ are serially generated, afterwards parallelized and then mapped with On-Off-Keying (OOK) onto the $N_{\text {sub }}$ engendered subband pulses.

In addition, the system's flexibility with respect to different aspects such as the scalability of data rates, the avoidance of narrowband interference [8] or the adaptation to arbitrary regulation masks is increased via an internal one-dimensional bandplan $L=\left[l_{1}, \ldots, l_{N_{\text {sub }}}\right]$ of size $N_{\text {sub }}$, which indicates deactivated subbands with a binary 0 and activated subbands with a binary 1 , respectively. Hence, the number of active marked subbands conducts to $\sum_{i=1}^{N_{\text {sub }}} l_{i}$. Finally, the modulated subband pulses $p_{i}(t), i \in\left\{1, \ldots, N_{\text {sub }}\right\}$ of the activated subbands are added up, leading to the transmit multiband signal

$$
s(t)=\sum_{n=-\infty}^{\infty} \underbrace{\sum_{i=1}^{N_{\text {sub }}} b_{i, n} l_{i} p_{i}\left(t-n T_{\mathrm{r}}\right)}_{\tilde{p}_{n}(t)} .
$$

In the above equation the inner sum $\tilde{p}_{n}(t)$ describes one complete multiband symbol. Each multiband symbol is sent according to the pulse repetition time $T_{\mathrm{r}}$, which has to be larger or equal the pulse duration $T_{\mathrm{p}}$ to reduce intersymbol interference. 


\section{B. Receiver Structure}

On the receiver, the transmitted signal $s(t)$ is superimposed with noise $n(t)$ which is modeled as additive white gaussian noise (AWGN) with two-sided spectral noise density $N_{0} / 2$. The received signal

$$
y(t)=s(t)+n(t),
$$

is separated by a bandplan-controlled analog band-pass filter bank, leading to the received subband pulses $y_{i}, i \in$ $\left\{1, \ldots, N_{\text {sub }}\right\}$ of the activated subbands. On each activated subband a non-coherent energy detection, consisting of a squaring and an integrator device with intergration time $T_{\mathrm{i}}$ is implemented. Thereby, two hypotheses, depending on whether noise plus signal or noise only has been detected, are possible for the received signal energy $x$. The decision between both hypotheses is based on a maximum-likelihood (ML) detection, comparing the received signal energy with a system specific threshold. It can be shown that the optimum threshold results from the intersection of a central and a non-central $\chi^{2}$ probability density function [9]. However, as the optimum threshold is hard to find, we choose an effective low complexity approximation by considering an adaptive tabulated function which depends on recursive estimated signal energy and noise power density [5].

Eventually, the important MIR-UWB system parameters used in this paper are summarized: We are considering an MIR-UWB system with a maximal subband number $N_{\text {sub }}=$ 24 . Within pulse repetition time $T_{\mathrm{r}}=50 \mathrm{~ns}$, pulse generation, which is based on filter bank pulses of pulse duration $T_{\mathrm{p}}=$ $15 \mathrm{~ns}$, is regularly done in every subband. Thereby, the used filter bank consists of elliptic filters of order $n_{\mathrm{f}}=4$, a subband bandwidth $B_{\text {sub }}=312.5 \mathrm{MHz}$ and subband frequency offset $B_{\text {off }}=62.5 \mathrm{MHz}$. The pulses are modulated with OOK and transmitted via an AWGN channel. Finally, the non-coherent receiver is based on a synchronization resolution of $1 \mathrm{~ns}$ and an integration time $T_{\mathrm{i}}=25 \mathrm{~ns}$. Considering such an MIRUWB system, the maximum total uncoded data rate conducts to $480 \mathrm{Mbit} / \mathrm{s}$.

\section{INTERFERENCE IMPACT ON THE MIR-UWB SYSTEM}

This section illustrates the impact of an interferer to the MIR-UWB system without implementing any mitigation strategy.

\section{A. Interference source: $M B-O F D M U W B$}

One important interference source for future high-speed wireless MIR-UWB definitely will be MB-OFDM UWB [1], resulting from the fact that first products are already on the market. MB-OFDM UWB unifies conventional OFDM with a multiband approach, whereas the total available spectrum of $7.5 \mathrm{GHz}$ is subdivided into 14 subbands of bandwidth $B_{\mathrm{OFDM}}=528 \mathrm{MHz}$ which are combined to 5 band groups. Due to the fact that first devices only operate on the 3 bands of band group 1 and that the remaining band groups are optional, we assume in this paper that the interference source operates only on band group 1 .

Within band group 1, the QPSK modulated OFDM symbols with symbol duration $T_{\text {OFDM }}=312.5 \mathrm{~ns}$ are transmitted in a time-interleaved manner. Thereby, unlike traditional OFDM systems, MB-OFDM UWB offers a channel segmentation mechanism to minimize mutual interferences of devices from different spatially overlapping uncoordinated piconets which use the same transmission media at the same time. For this

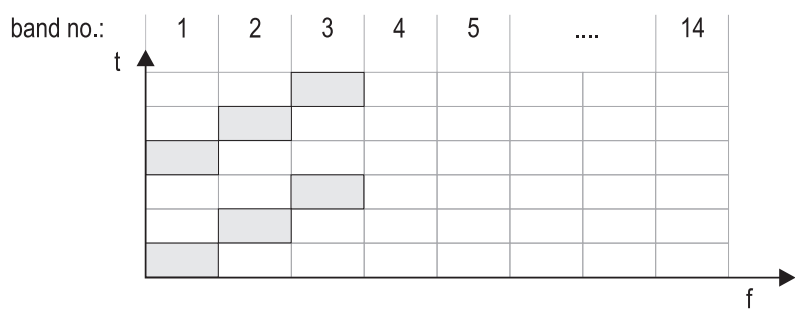

Fig. 1. Considered time-frequency code

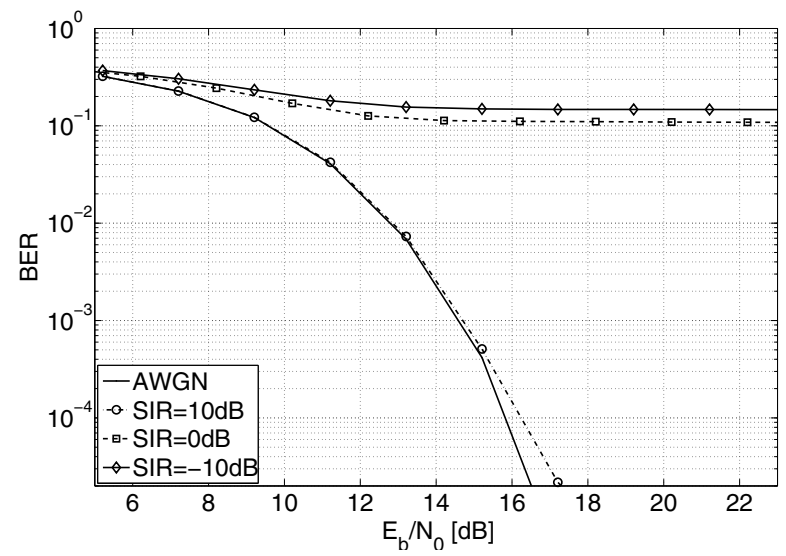

Fig. 2. BER vs. $E_{b} / N_{0}$ for weak $(\mathrm{SIR}=10 \mathrm{~dB})$, mean $(\mathrm{SIR}=0 \mathrm{~dB})$ and strong $(\mathrm{SIR}=-10 \mathrm{~dB})$ interference

reason different characteristic time-frequency codes, ensuring frequency diversity on one side and allowing multiple access between different piconets on the other side, are allocated to each operating piconet. The time-frequency code describes the sequence of subband center frequencies changes after each OFDM symbol. In this paper we assume only one operating interferer with a time-frequency code $(1,2,3,1,2,3, \ldots)$ which is illustrated in Fig. 1.

\section{B. Simulation Results: Interference Impact}

To illustrate the interferer's impact, it is assumed that the MB-OFDM UWB interference source is always present and that all bands of the MIR-UWB system are active during the whole time. Then, the signal-to-interference ratio (SIR) is given by

$$
\mathrm{SIR}=10 \log _{10}\left(\frac{P_{\tilde{p}}}{P_{\mathrm{OFDM}}}\right),
$$

whereas $P_{\tilde{p}}$ and $P_{\text {OFDM }}$ stand for the mean signal power of a multiband pulse and an MB-OFDM UWB signal with respect to the total bandwidth of $7.5 \mathrm{GHz}$.

In Fig. 2 the bit error rate (BER) is plotted vs. $E_{b} / N_{0}$ for a strong $(\mathrm{SIR}=-10 \mathrm{~dB})$, a mean $(\mathrm{SIR}=0 \mathrm{~dB})$ as well as a weak (SIR $=10 \mathrm{~dB}$ ) interference impact. It is obvious that interference within the mean and strong region leads to a tremendous decrease of the MIR-UWB system's performance. Only for weak interference the influence to the MIR-UWB system is minor.

To further characterize the interferer's impact on the MIRUWB system, Fig. 3 shows the BER vs. varied SIR values for two fixed $E_{b} / N_{0}$ values ( $11 \mathrm{~dB}$ and $15 \mathrm{~dB}$ ). For both $E_{b} / N_{0}$ values, an asymptotic approximation of the BER curve to the BER of the interference-free case can be observed. E.g., the 


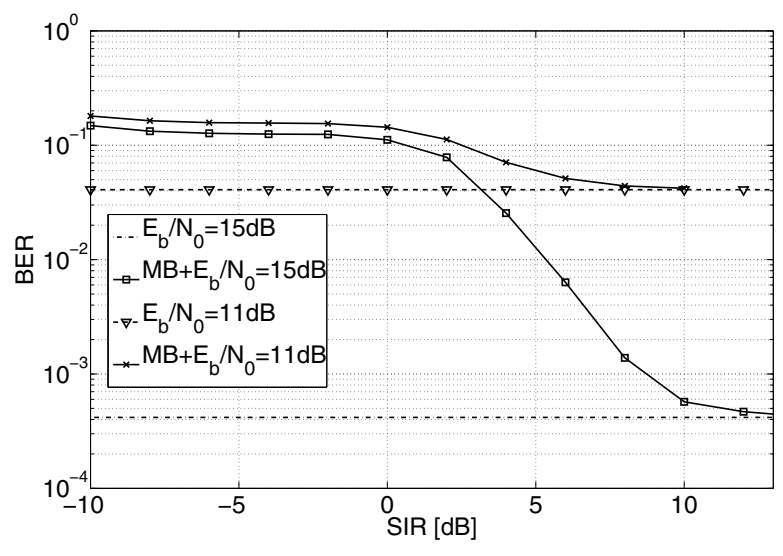

Fig. 3. BER vs. SIR for fixed $E_{b} / N_{0}(11 \mathrm{~dB}$ and $15 \mathrm{~dB})$

interference influence is negligible for a SIR value of $6 \mathrm{~dB}$ (this corresponds to a relative distance $d_{r e l}=d_{O F D M} / d_{M I R} \approx 2$ under free space path loss, whereas $d_{M I R}$ equals the MIR transmitter-MIR receiver distance and $d_{O F D M}$ equals the MBOFDM transmitter-MIR receiver distance) for $E_{b} / N_{0}=11 \mathrm{~dB}$ and $10 \mathrm{~dB}\left(d_{r e l} \approx 3.16\right)$ for $E_{b} / N_{0}=15 \mathrm{~dB}$.

\section{Coexistence ApProAch}

\section{A. Motivation}

As shown in the previous section, the impact of an MBOFDM UWB interference source can be so dominant that communication within an uncoded MIR-UWB system is not possible at all. That's why low complex, efficient and robust coexistence techniques are necessary for the MIR-UWB system without changing its system configuration. In this context, we will distinguish between a static as well as an adaptive coexistence approach.

The static coexistence approach is the solution with lowest complexity. It is based on a complete deactivation of possibly interfered subbands via the MIR-UWB's bandplan before any data transmission takes place. In that case best BER performance can be achieved without spending effort into interference detection or mitigation. However, a complete deactivation of subbands leads to a loss with respect to maximum achievable data rates and is unacceptable, inflexible with respect to dynamic interference situations. That's why an efficient and dynamic adaptive coexistence approach is proposed in the next subsection, for which the static approach will be used as a reference.

\section{B. Adaptive Coexistence}

The impact of interference can be handled when taking the specific time-frequency code of the MB-OFDM UWB interferer into account. For this reason, an adaptive coexistence approach is integrated into the basic initialization method which is introduced by Paquelet and Aubert [5].

This method is extended to initially estimate the necessary parameters, the bit energy $E$ as well as the noise power density $N$, for each demodulator separately in every activated subband $i \in\left\{1, \ldots, N_{\text {sub }}\right\}$. It is realized via a preamble, containing $m_{0}$ binary zeros and $m_{1}$ binary ones $\left(m_{0}+m_{1}=2000\right)$, which are transmitted over each subband $i$ in parallel. Then, based on the maximum-likelihood (ML) method, an unbiased estimation of the noise power density $N_{i}$ is conducted in each subband $i$, leading to

$$
\hat{N}_{i}=\frac{\sum_{j=1}^{m_{0}} x_{j, i}}{m_{0} M},
$$

whereas the variance of this estimator is $\sigma_{\hat{N}_{i}}^{2}=\frac{N_{i}^{2}}{m_{0} M}$. In a further step, based on the aforementioned initial estimated noise power densities $\hat{N}_{i}$, an initial biased estimation of bit energies $E_{i}$

$$
\hat{E}_{i}=\frac{\sum_{j=m_{0}+1}^{m_{0}+m_{1}} x_{j, i}}{m_{1}}-M \hat{N}_{i},
$$

is done in every subband $i$. The variance of this estimator is given as $\sigma_{\hat{E}_{i}}^{2}=\frac{M N_{i}^{2}+2 E_{i} N_{i}}{m_{1}}+M^{2} \sigma_{\hat{N}_{i}}^{2}$. In both equations $x_{j, i}$ describes the $j^{\text {th }}$ received energy value in subband $i$ and the parameter $M$ stands for the space dimensionality of a finite energy signal of effective subband bandwidth $B_{\text {eff }}$, which is observed over integration time $T_{\mathrm{i}}[9]$.

In addition to this initial channel estimation, the same $m_{0}$ binary zeros which are sent over each subband $i$ can be simultaneously used for interference detection within each subband. In this context a global clustering strategy, the method of Otsu, can be applied [10]. Thereby, the total $m_{0} N_{\text {sub }}$ received energy values can be interpreted as a time-frequency pattern, whereas each pattern element corresponds to a gray level associated pixel. In this case a gray level can be seen as a specific energy potential within the received energy values.

To find a threshold $\epsilon_{t h}$, which can be used for the decision of interfered or not interfered pixels, it is assumed that $K \geq$ $m_{0} N_{\text {sub }}$ energy potentials $E_{\min }=\epsilon_{1} \leq \ldots \leq \epsilon_{K}=E_{\max }$ are equally distributed in increasing order over the observed energy value interval $\left[E_{\min } ; E_{\mathrm{max}}\right]$ (Fig. 4a). Thereby, $E_{\min }$ stands for the minimum energy value within the present time-frequency pattern and $E_{\max }$ for the maximum value, respectively.

By allocating energy values to their nearest energy potential $\epsilon_{i}$, an energy potential distribution, which is described via

$$
p_{i}=\frac{n_{i}}{m_{0} N_{\text {sub }}}, i \in 1, \ldots, K,
$$

results. To obtain a separation between interfered and not interfered energy values, two energy classes $C_{0}$ and $C_{1}$ are considered. Energy class $C_{0}$ contains not interfered energy values with indices $1, \ldots, k$, whereas energy class $C_{1}$ comprises interfered energy values with indices $k+1, \ldots, K$. In Fig. 4a the initial index $k$ is set to the arithmetic mean of lowest and highest occuring energy potential $k=\lfloor K / 2\rfloor$. Based on that arrangement two optimization possibilities can be conducted.

The first optimization option consists of the minimization of the combined empirical energy class variance (Fig. 4b), which can be defined as

$$
s_{w}^{2}(k)=s_{C_{0}}^{2}(k) P_{C_{0}}(k)+s_{C_{1}}^{2}(k) P_{C_{1}}(k) .
$$

This step aims at an adjustment of the overall initially set index $k$, whereas a correct allocation of energy outliers to the corresponding other class is achieved. In the above expression, $P_{C_{0}}(k)$ and $P_{C_{1}}(k)$ stand for the occurence probabilities

$$
P_{C_{0}}(k)=\sum_{i=1}^{k} p_{i}, \quad P_{C_{1}}(k)=1-P_{C_{0}}(k),
$$




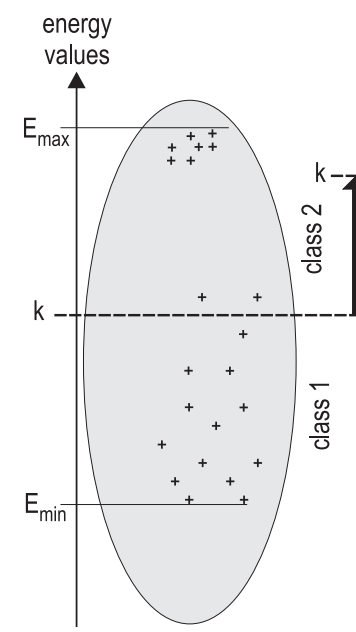

a)

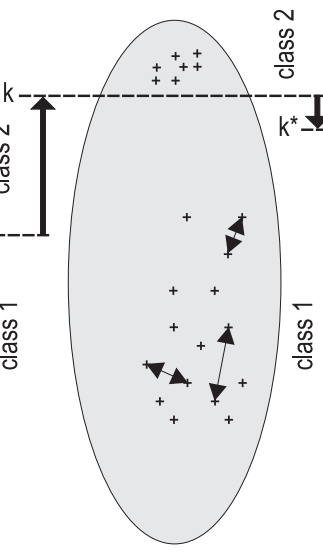

b)

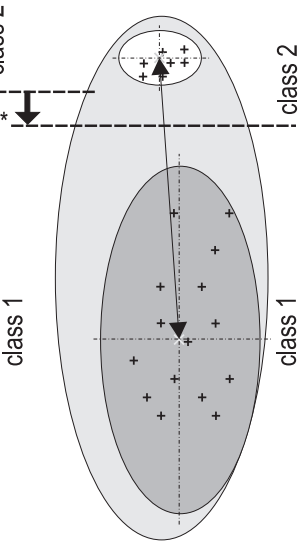

c)

Fig. 4. Threshold determination

of energy classes $C_{0}$ and $C_{1}$. Furthermore, we used the empirical energy class mean levels

$$
\bar{x}_{C_{l}}(k)= \begin{cases}\sum_{i=1}^{k} \frac{\epsilon_{i} p_{i}}{P_{C_{l}}(k)}, & l=0 \\ \sum_{i=k+1}^{K} \frac{\epsilon_{i} p_{i}}{P_{C_{l}}(k)}, & l=1\end{cases}
$$

describing the class means as well as the empirical energy class variances

$$
s_{C_{l}}^{2}(k)= \begin{cases}\sum_{i=1}^{k}\left(\epsilon_{i}-\bar{x}_{C_{l}}(k)\right)^{2} \frac{p_{i}}{P_{C_{l}}(k)}, & l=0 \\ \sum_{i=k+1}^{K}\left(\epsilon_{i}-\bar{x}_{C_{l}}(k)\right)^{2} \frac{p_{i}}{P_{C_{l}}(k)}, & l=1 .\end{cases}
$$

The second optimization criterion considers the maximization of the empirical variance between both energy classes, which can be defined as

$$
\begin{aligned}
s_{b}^{2}(k)= & \left(\bar{x}_{C_{0}}(k)-\bar{x}_{t o t}\right)^{2} P_{C_{0}}(k) \\
& +\left(\bar{x}_{C_{1}}(k)-\bar{x}_{t o t}\right)^{2} P_{C_{1}}(k),
\end{aligned}
$$

whereas the total empirical mean value $\bar{x}_{t o t}$ of the overall time-frequency pattern is given as $\bar{x}_{t o t}=\bar{x}_{C_{0}}+\bar{x}_{C_{1}}$ (Fig. 4c). Hence, a separation of both classes with respect to the mean value of the total time-frequency pattern is obtained, leading to a more accurate adaptation of index $k$.

As both optimization criteria have opposing effects with respect to the best index $k^{\star}$, they are combined into one characteristic optimization criterion which is defined as [10]

$$
k^{\star}=\arg \max _{k=1, \ldots, K} \frac{s_{b}^{2}(k)}{s_{w}^{2}(k)} .
$$

According to the calculated index $k^{\star}$, corresponding to threshold $\epsilon_{t h}=\epsilon_{k^{\star}}$, we decide binary for each received noise energy value $x_{j, i}, j=1, \ldots, m_{0}, i=1, \ldots, N_{\text {sub }}$ and define a cell as interfered if $x_{j, i} \geq \epsilon_{t h}$.

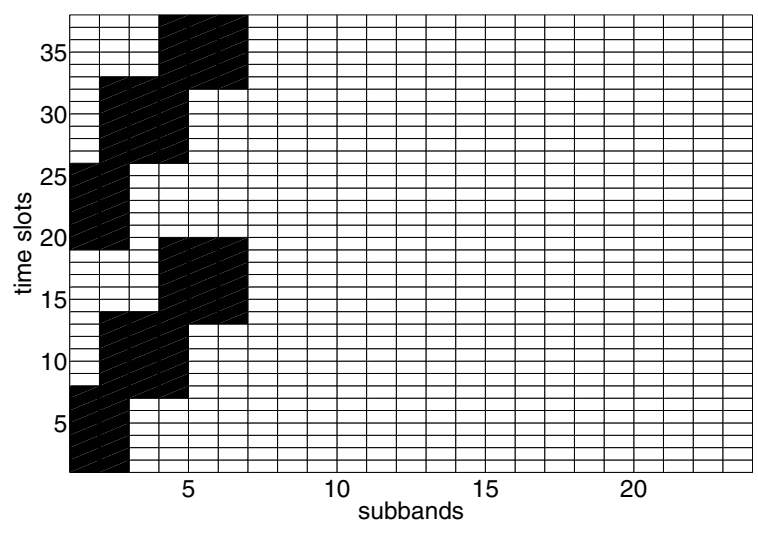

Fig. 5. Exemplary generation of two-dimensional bandplan array, $E_{b} / N_{0}=$ $15 \mathrm{~dB}, \mathrm{SIR}=-10 \mathrm{~dB}$. C.f. also the interferer time-frequency code in Fig.1

\section{2-dimensional Bandplan}

Doing the above mentioned binary decision of subsection IV-B for all energy values, a two-dimensional bandplan array $L^{\star}=\left[L_{1} ; L_{2} ; \ldots ; L_{m_{0}}\right]$ of size $m_{0} \times N_{\text {sub }}$, containing $m_{0}$ realizations of the one-dimensional bandplan is generated. As $L^{\star}$ covers several MB-OFDM UWB symbols, an increase of the demarcation of interfered and not interfered energy regions is possible via a combined segmentation and overlap procedure which requires knowledge of the interferer's periodic behavior. This assumption is based on the fact, that knowledge of characteristic interference properties such as the interferer's symbol duration or periodicity of to expected interference sources is reasonable in practical applications.

After the segmentation and overlap process a modified twodimensional bandplan array of the interferer's periodicity size results which is then used for the initial estimation of signal energy in (5). Fig. 5 shows one exemplary generated bandplan for a fixed $E_{b} / N_{0}$ of $15 \mathrm{~dB}$ and a fixed SIR of $-10 \mathrm{~dB}$ from the MIR's point of view. Each small box characterizes an energy value at an arbitrary time and frequency. As the MIR's pulse repetition time is approximately $T_{\mathrm{OFDM}} / T_{\mathrm{r}}=6.25$ times smaller than the MB-OFDM UWB symbol duration up to 7 not plausible energy values can occur within one subband. Furthermore, due to the MIR's smaller subband bandwidth of $B_{\text {OFDM }} / B_{\text {eff }}=2.11$ up to 3 subbands can be simultaneously interfered. In this context it should be notified that there exist mixed and full interfered subbands which could influence the algorithms performance. It is assumed that the receiver transmits this two-dimensional bandplan array after each periodically repeated initialization phase to the transmitter via an error free feedback channel. Then, the transmitter allocates bits in accordance to that bandplan up to the next initialization phase, independent of whether the interferer disappears during data transmission phase. Hence, according to the specific interference situation, the MIR system's subband configuration is adapted, contributing to improved system performance.

\section{Simulation Results}

As we are mainly interested in high data rate communication this approach allows to send data during not interfered time gaps while maintaining the system performance. This is illustrated in Fig. 6 for a fixed working point of $15 \mathrm{~dB}$. Thereby, a comparison of data rate with respect to different 


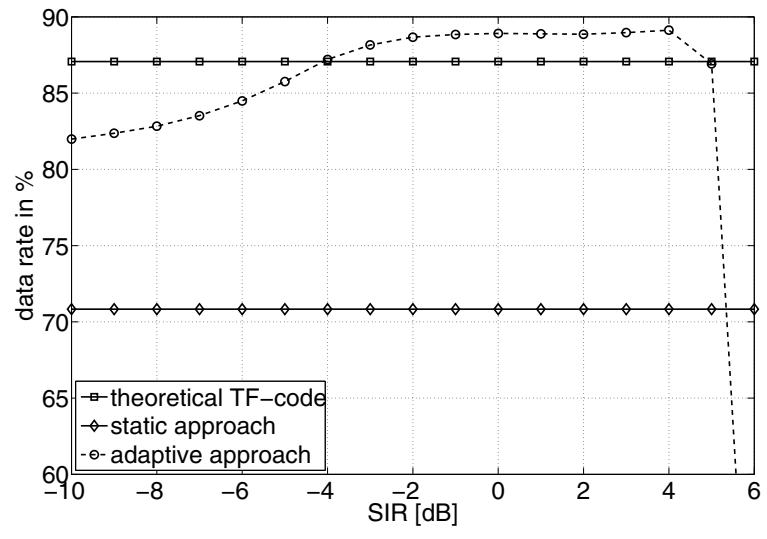

Fig. 6. Comparison of data rate vs. SIR for a fixed $E_{b} / N_{0}$ of $15 \mathrm{~dB}$ for static approach, theoretical time-frequency code and adaptive approach

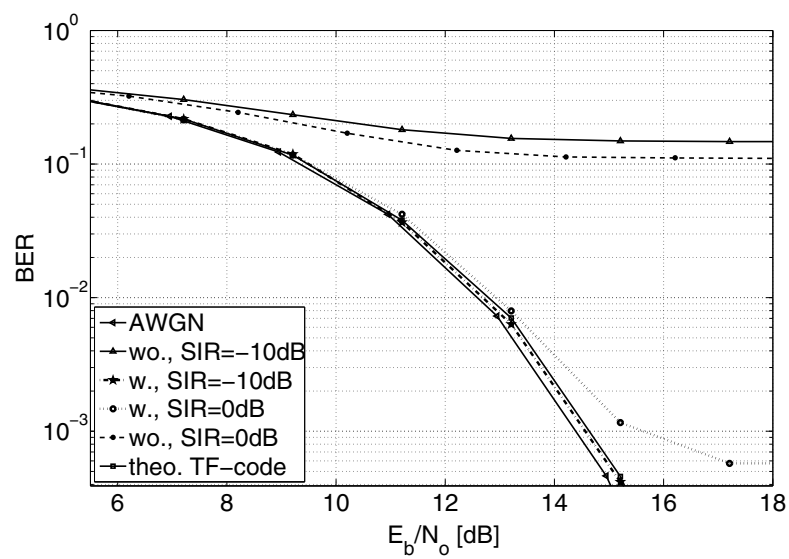

Fig. 7. BER vs. $E_{b} / N_{0}$ for weak $(\mathrm{SIR}=0 \mathrm{~dB})$ and strong $(\mathrm{SIR}=-10 \mathrm{~dB})$ interference w/wo adaptive approach, reference: theoretical time-frequency code, AWGN

strong interference is illustrated for the static approach, the adaptive approach as well as the theoretically optimal case.

In contrast to the static approach the adaptive approach can allow significant higher data rates which can also be higher than the reference for the case of optimal interference related bandplan setting. Furthermore, it is recognizable that for practical applications, the adaptive method only works well up to an SIR value of approximately $4 \mathrm{~dB}$. Then, for higher SIR a drastic decay of data rate up to nearly $0 \%$ will occur. Thereby, more decisions for interfered energy values are made due to the diminish differences of both energy classes. E.g., minimum data rate means that the adaptive approach interpretes the weakest energy value as just not interfered and all the other energy values as interfered. That characteristic behavior can be used for activation or deactivation of the adaptive approach. Finally, for SIR values higher than that transition range data rate will increase to approximately $50 \%$ because the algorithm makes quasi arbitrary decisions.

On the other side, the higher achievable data rates of the pure application of the adaptive approach influences the BER performance. This is illustrated in Fig. 7, where the BER is plotted vs. $E_{b} / N_{0}$ for weak (SIR $=0 \mathrm{~dB}$ ) and strong $(\mathrm{SIR}=-10 \mathrm{~dB})$ interference. The AWGN curve, which repre- sents the static approach serves as lower bound. The advantage of the presented adaptive approach can be clearly observed, as there's a significant gain compared to the worst case scenario of no interference coexistence. However, it is obvious, that for weak interference $(\mathrm{SIR}=0 \mathrm{~dB})$, the pure application of the adaptive approach leads to a performance loss which appears in an error floor. The reason for the occuring error floor lies in the influence of wrong detected mixing bands with reduced power levels. The adaptive algorithm works better for stronger interference (SIR $=-10 \mathrm{~dB}$ ), for which the lower bound is almost reached. However, as best system performance has to be judged under both aspects, data rate and BER performance, best performance will be achieved when having regard to the standard related time-frequency bandplan setting.

\section{COnClusions}

This paper analyzes the impact of MB-OFDM UWB, operating in band group 1 with a specific periodic timefrequency code, on a non-coherent MIR-UWB system. Two different coexistence approaches for interference mitigation are investigated. A static approach is very efficient and can be realized with lowest complexity. However, as system capacity in the context of data rate is significantly decreased, a second low complex adaptive approach, aiming at an increased flexibility with respect to data rate and interference occurence, is presented. Thereby, a two-dimensional bandplan can be used to fill up idle time-frequency gaps during data transmission. Performance investigations show the efficiency and robustness of the adaptive approach in the context of data rate and BER performance for mean and strong interference.

The modified MIR-UWB architecture with its low complex and efficient coexistence algorithm can be realized with minor hardware changes and is applicable under different regulations for low power and low cost high data rate applications.

\section{ACKNOWLEDGMENTS}

The authors gratefully acknowledge that their work is supported within the priority program No. 1202/2 (UKoLoS) by the German Research Foundation (DFG).

\section{REFERENCES}

[1] A. Batra, J. Balakrishnan et al., "Multiband OFDM physical layer proposal for IEEE 802.15 task group 3a," Sep. 2004.

[2] Federal Communications Commission, "First report and order," ET Docket No. 98-153, Tech. Rep., April 22, 2002.

[3] M. Win and R. Scholtz, "Impulse radio: how it works," IEEE Communication Letters, vol. 2, no. 2, pp. 36-38, Feb. 1998.

[4] L. M. Aubert, "Mise en place d'une couche physique pour les futurs systèmes de radiocommunications hauts débits UWB," Ph.D. dissertation, Nov. 2005

[5] S. Paquelet and L. M. Aubert, "An Energy Adaptive Demodulation for High Data Rates with Impulse Radio," in RAWCOM, Atlanta, USA, Sep. 2004.

[6] S. Paquelet, L. M. Aubert, and B. Uguen, "An Impulse Radio Asynchronous Transceiver for High Data Rates," in Proceedings of Joint UWBST \& IWUWBS, Kyoto, Japan, May 2004.

[7] M. Mittelbach, R. Moorfeld, and A. Finger, "Performance of a Multiband Impulse Radio UWB Architecture," in 3rd IEE Mobility Conference, Bangkok, Thailand, Oct. 2006.

[8] R. Moorfeld, A. Finger, H. Dehner, H. Jäkel, and F. K. Jondral, "A simple and fast detect and avoid algorithm for non-coherent multiband impulse radio UWB," in ISSSTA2008, Bologna, 2008.

[9] P. A. Humblet and M. Azizoglu, "On the bit-error rate of lightwave systems with optical amplifiers," Journal of Lightwave Technology, vol. 9, pp. 1576-1582, 1991.

[10] N. Otsu, "A Threshold Selection Method from Gray-Level Histograms," IEEE Transactions on Systems, Man, and Cybernetics, vol. 9, pp. 62-66, 1979. 